\title{
The predictive role of haematological parameters in the diagnosis of osteoarticular brucellosis
}

\author{
Şafak Özer Balın, Ayşe Sağmak Tartar, Ayhan Akbulut
}

Frrat University Medical Faculty Hospital, Department of Infectious Disease, Elazig, 23000, Turkey

\section{Author details:}

Şafak Özer Balın, Tel: +90-233-35-55: E-mail: safakozerbalin@hotmail.com;

Ayşe Sağmak Tartar, Tel: +90-233-35-55: E-mail: dr.ayse01@gmail.com;

Ayhan Akbulut,Tel: +90-233-35-55: E-mail: a_akbulut@yahoo.com

\begin{abstract}
Background: Brucellosis is a zoonosis that affects several systems, especially with the osteoarticular involvement.

Objectives: This study aims to compare the neutrophil/lymphocyte ratio (NLR), platelet/lymphocyte ratio (PLR), monocyte/ lymphocyte ratio (MLR), C-reactive protein (CRP), erythrocyte sedimentation rate (ESR), mean platelet volume (MPV) and red blood cell distribution (RDW) in patients with the osteoarticular involvement and those with non-localised brucellosis and evaluate their predictive value for the diagnosis of osteoarticular brucellosis.

Methods: We enrolled 140 patients with brucellosis, 70 with the osteoarticular involvement and 70 without any localised involvement. We collected patients' data retrospectively and compared haematological parameters between both groups. In patients with osteoarticular brucellosis, a correlation of the NLR with the ESR and CRP and correlation of the MLR with the ESR and CRP were assessed. Furthermore, the predictive performance of the ESR, CRP, NLR and MLR on the osteoarticular involvement was evaluated.

Results: The NLR, MLR, ESR, CRP, neutrophil and monocyte levels were higher in the patient group than the control group. Conclusion: The NLR, MLR, ESR and CRP are useful parameters to estimate the clinical course of patients with brucellosis, and the NLR and MLR are alternative to inflammatory markers in the osteoarticular involvement.
\end{abstract}

Keywords: Neutrophil/lymphocyte ratio, monocyte/lymphocyte ratio, inflammatory marker.

DOI: https://dx.doi.org/10.4314/ahs.v18i4.19

Cite as: Baln ŞÖ, Tartar AS, Akbulut A. The predictive role of haematological parameters in the diagnosis of osteoarticular brucellosis. Afri Health Sci. 2018;18(4): 988-994. bttps:// dx.doi.org/10.4314/ahs.v18i4.19

\author{
Corresponding author: \\ Şafak Özer Balın, \\ F1rat University Medical Faculty Hospital, \\ Department of Infectious Disease, \\ Elazig, 23000, Turkey \\ Tel: +90-233-35-55 \\ E-mail: safakozerbalin@hotmail.com
}

\section{Introduction}

Brucellosis is a common zoonosis prevalent around the world ${ }^{1}$ and is reported in humans with low mortality but high morbidity. Thus, it remains a critical public health problem ${ }^{2,3}$. As brucellosis affects various organs and tissues, its clinical course and severity are variable ${ }^{4}$ Occasionally, it is considered a localised infection ${ }^{5}$. Localised infections, mostly osteoarticular, involve haematological, 
genitourinary, gastrointestinal, cardiovascular and neurological systems ${ }^{6}$. The disease is diagnosed with serological test or culture positivity together with clinical findings ${ }^{7}$. However, laboratory conditions might not always be appropriate or adequate for the diagnosis. Hence, it might be beneficial to use additional diagnostic methods. Leukocyte count and C-reactive protein (CRP) are among the leading infection markers used in brucellosis cases. High neutrophils and lymphocytes are closely associated with certain inflammatory conditions. In addition, platelets have been reported to be associated with inflammation $^{8}$. Recently, leucocyte sub-group ratios have been used as markers in various diseases ${ }^{9,10}$. Moreover, some studies have investigated whether the mean platelet volume (MPV) and red blood cell distribution (RDW) values could be accepted as indicators ${ }^{11,12}$. The MPV is associated with the platelet activation and function and has been reported as an inflammatory marker in some diseases ${ }^{13}$, which demonstrates the presence of the inflammatory burden and disease activity in several diseases, including pre-eclampsia, acute pancreatitis, myocardial infarction and systemic inflammatory conditions like ulcerative colitis and Crohn's disease ${ }^{14}$. Reportedly, the RDW is a predictive value in inflammatory and infectious pathologic diseases, including inflammatory bowel disease, celiac disease, pulmonary embolism, coronary artery disease, acute pancreatitis, bacteremia, sepsis and septic shock ${ }^{15}$. Nevertheless, the properties of the MPV, RDW and leucocyte sub-group ratios specific for patients with osteoarticular brucellosis remain partially elucidated.

This study aims to compare the neutrophil/lymphocyte ratio (NLR), platelet/lymphocyte ratio (PLR), monocyte/ lymphocyte ratio (MLR), CRP, erythrocyte sedimentation rate (ESR), MPV and RDW in patients with the osteoarticular involvement and those with non-localised brucellosis and evaluate their predictive value for the diagnosis of osteoarticular brucellosis. Most studies in this group of adult patients were conducted to compare haematological parameters between patients with brucellosis and the healthy control group.

\section{Methods}

In this study, we enrolled 140 patients with brucellosis, 70 with the osteoarticular involvement (patient group) and 70 without any localised involvement (control group), who visited the Infection Diseases Department of Firat
University Medical Faculty Hospital during 2012-2017. The demographic data and laboratory values of all patients were obtained retrospectively from the ENLIL system of our hospital. Brucellosis was diagnosed with Brucella bacteremia in blood culture or the positive Wright test and/or Wright with Coombs test in the presence of clinical findings without any bacteremia. Notably, patients diagnosed with brucellosis and also sacroiliitis and/or spondylodiscitis by radiological imaging were included in the patient group with the osteoarticular involvement.

In contrast, patients with brucellosis with no localised involvement (haematological, genitourinary, gastrointestinal, cardiovascular and neurological system involvement) were assigned to the control group. The exclusion criteria were as follows: patients with a chronic inflammatory disease, severe liver, kidney and cardiac disease or another infectious disease, patients aged $<18$ years, or patients admitted to our hospital under treatment. We measured haematological parameters, such as the neutrophil count, lymphocyte count, monocyte count, platelet count, CRP, ESR, RDW and MPV, for both groups. In addition, the NLR, MLR and PLR were evaluated as the ratio of neutrophils-to-lymphocytes, monocytes-to-lymphocytes and platelets-to-lymphocytes, respectively. We compared haematological parameters between both groups. Moreover, a correlation of the NLR with the ESR and CRP and a correlation of the MLR with the ESR and CRP were assessed in patients with osteoarticular brucellosis. Furthermore, the predictive performance of the ESR, CRP, NLR and MLR on the osteoarticular involvement was evaluated. This study protocol was approved by the Ethics Committee on non-invasive clinical investigations of the Faculty of Medicine of Firat University on 4 April 2018.

\section{Statistical analysis}

In this study, the Kolmogorov-Smirnov/Shapiro-Wilk test was performed to control the normal distribution of variables. Data are presented as mean \pm standard deviation for continuous variables with normal distribution and as median (median; minimum-maximum) for data without normal distribution. In addition, categorical variables are presented as frequency and percentage. Using the Mann-Whitney U-test or Student's t-test to compare continuous variables depending on the normality of the data distribution. However, one-way ANOVA or Kruskal-Wallis tests were used for multiple comparisons. We 
determined the correlation between two continuous variables using the Spearman correlation test. Furthermore, cut-off values of the parameters used to differentiate between patients with and without the osteoarticular involvement were evaluated using the receiver-operating characteristic (ROC) analysis. We considered $\mathrm{P}<0.05$ as statistically significant. The data analysis in this study was performed using the SPSS-22 package programme.

\section{Results}

In this study, we enrolled 140 patients (76 [54.3\%] males and $64[45.7 \%$ ] females; mean age: $46.5 \pm 20.1$ [range: 19-85] years; median: 46 years) with brucellosis, 70 with the osteoarticular involvement (patient group) and 70 without any local involvement (control group). The age and gender of patients in the patient group were matched to the age and sex of patients in the control group $(\mathrm{P}>$ 0.05; Table 1). The NLR, MLR, ESR, CRP, neutrophil and monocyte levels were significantly higher in the patient group than the control group $(\mathrm{P}<0.05)$.

Table 1. Laboratory results of patients with osteoarticular brucellosis (patient group) and patients with brucellosis without the osteoarticular involvement (control group)

\begin{tabular}{llll}
\hline & Patients & Controls & $P$ \\
\hline Gender $($ male/female $)$ & $39 / 31$ & $37 / 33$ & $>0.05$ \\
Age $($ year $)$ & $43.9 \pm 22.8$ & $50 \pm 15.9$ & $>0.05$ \\
Neutrophil count $\left(\times 10^{9} / \mathrm{L}\right)$ & $4690(2090-8450)$ & $2505(1320-4010)$ & $<\mathbf{0 . 0 5}$ \\
Lymphocyte count $\left(\times 10^{9} / \mathrm{L}\right)$ & $2357 \pm 824$ & $1958 \pm 743$ & $>0.05$ \\
Monocyte count $\left(\times 10^{9} / \mathrm{L}\right)$ & $515(50-5480)$ & $380(100-1000)$ & $<\mathbf{0 . 0 5}$ \\
Platelet count $\left(\times 10^{9} / \mathrm{L}\right)$ & $307904 \pm 69695$ & $285125 \pm 127016$ & $>0.05$ \\
HGB $(\mathrm{g} / \mathrm{L}$ & $13(8,9-16,4)$ & $13,8(6,6-15,9)$ & $>0.05$ \\
NLR & $1.89(0.71-12.5)$ & $1.4(0.76-2.7)$ & $<\mathbf{0 . 0 5}$ \\
MLR & $0.25(0.04-2.19)$ & $0.21(0.09-0.33)$ & $<\mathbf{0 . 0 5}$ \\
PLR & $116.5(66-300)$ & $131.9(79-304)$ & $>0.05$ \\
MPV $(\mathrm{fL})$ & $7.9 \pm 0.9$ & $8.5 \pm 1.2$ & $>0.05$ \\
RDW $(\%)$ & $15.5 \pm 2.6$ & $14.6 \pm 2.2$ & $>0.05$ \\
CRP $(\mathrm{mg} / \mathrm{L})$ & $32.1(3.2-84.2)$ & $5.5(3-45.8)$ & $<\mathbf{0 . 0 5}$ \\
ESR $(\mathrm{mm} / \mathrm{h})$ & $43(1-120)$ & $19(2-65)$ & $<\mathbf{0 . 0 5}$ \\
\hline
\end{tabular}

HGB: haemoglobin, NLR: neutrophil/lymphocyte ratio, MLR: monocyte/lymphocyte ratio, PLR: platelet/lymphocyte ratio; MPV: Mean Platelet Volume, RDW: Red Blood Cell Distribution, CRP: C-reactive protein, ESR: erythrocyte sedimentation rate

The correlation coefficients of the NLR with the ESR and CRP (r) were $0.243(\mathrm{P}=0.043)$ and $0.258(\mathrm{P}=$ 0.031), respectively. Additionally, the correlation coeffi- cients of the MLR with the ESR and CRP (r) were 0.342 $(\mathrm{P}=0.004)$ and $0.334(\mathrm{P}=0.005)$, respectively, in accordance with the Spearman analysis (Fig. 1). These results were significant. 

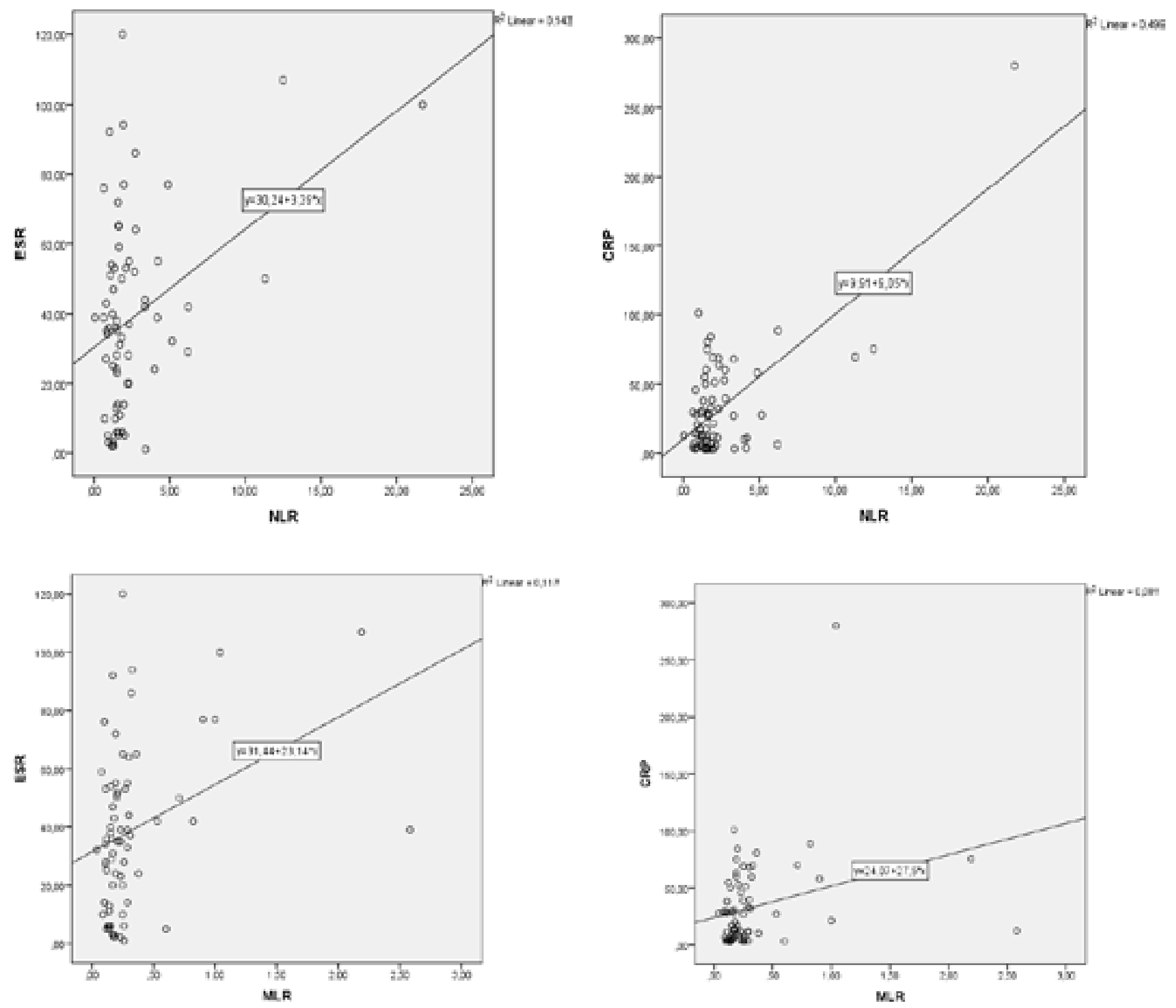

Figure 1. Scatter diagrams showing a correlation of the NLR with the ESR and CRP and of the MLR with the ESR and CRP in patients with osteoarticular brucellosis.

NLR: neutrophil/lymphocyte ratio, MLR: monocyte/lymphocyte ratio, CRP: C-reactive protein, ESR: erythrocyte sedimentation rate

In the patient group, the cut-off values of the ESR, CRP, NLR and MLR were 23.5, 26.6, 1.3 and 0.22, respectively (Fig. 2). The sensitivity and specificity were $70 \%$ and $60 \%$ for the ESR, $48 \%$ and $81 \%$ for the CRP, $70 \%$ and $51 \%$ for the NLR and $45 \%$ and $80 \%$ for the MLR, respectively. The area under the curve (AUC) of the ESR, CRP, NLR and MLR were $0.632,0.644,0.630$ and 0.604 , respectively. 


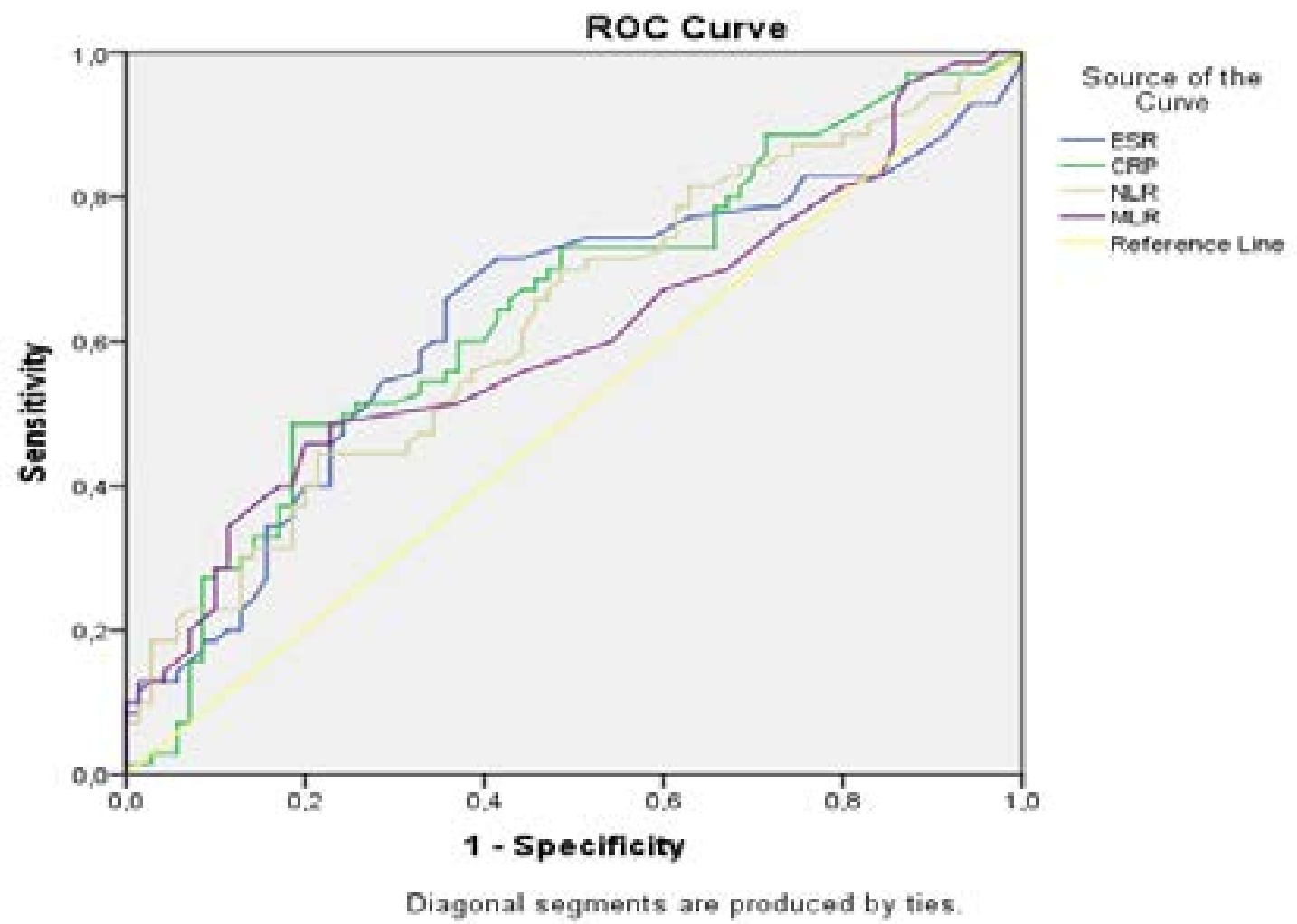

Figure 2. Estimation of the osteoarticular involvement in patients with brucellosis, ROC curve for the ESR, CRP, NLR and MLR ESR: erythrocyte sedimentation, CRP: C-reactive protein, NLR: neutrophil/lymphocyte ratio, MLR: monocyte/ymphocyte ratio

\section{Discussion}

Brucellosis is a common zoonotic systemic inflammatory disease that affects $>500,000$ people every year worldwide $^{16}$. As it affects all systems, it is often followed by non-specific manifestations, causing severe complications, primarily the osteoarticular involvement ${ }^{5}$. In these cases, delayed diagnosis and inappropriate treatment result in long-term morbidity in patients ${ }^{17}$. This study assessed the predictive role of haematological parameters in the diagnosis of patients with osteoarticular brucellosis.

The complete blood count, CRP and ESR are vital parameters in diagnosing brucellosis, determining the disease severity and assessing the treatment response in infectious and inflammatory diseases ${ }^{18}$. CRP is a non-specific parameter, which is closely associated with the prevalence of inflammation and disease progression. Togan et al. ${ }^{11}$ reported that the CRP level in patients with brucellosis was substantially higher than that in the control group. Another study reported that patients with osteoarticular brucellosis exhibited higher CRP levels than healthy group $^{12}$. In some studies, elevated ESR in brucellosis was closely related to the osteoarticular involvement ${ }^{19,20}$. In this study, CRP and ESR were significantly higher than that in the control group $(\mathrm{P}<0.05)$, which emphasises that both CRP and ESR are valuable markers in patients with osteoarticular brucellosis. During brucellosis, leucopenia, thrombocytopenia and lymphomonocytosis are also observed ${ }^{1,21}$. Neutrophil, monocyte, lymphocyte and platelet counts are complete blood count parameters that are readily available on a routine basis. Recent years have witnessed an increased interest in leucocyte sub-group ratios in reflecting the level of inflammation in some acute and chronic diseases ${ }^{22,23}$. Horne et al. ${ }^{24}$ reported that that NLR could be more effective than leucocyte levels in predicting inflammation. An increase in the NLR is considered an indicator of systemic inflammation ${ }^{25}$. In some studies, the NLR was considerably higher in patients with Behcet's disease $^{26}$, Sjogren's syndrome ${ }^{27}$, coronary artery disease $^{28}$ and tuberculosis spondylodiscitis ${ }^{29}$ than that in the control group. Aydin et al. ${ }^{30}$ reported that the MLR 
was considerably higher in patients with brucella epididymo-orchitis than in those with non-brucella epididymo-orchitis. In this study, patients with the osteoarticular involvement had higher levels of neutrophil, monocyte, NLR and MLR than that in the control group $(\mathrm{P}<0.05)$. Furthermore, we observed no statistically significant difference in the PLR. These findings suggest that neutrophil, monocyte, NLR and MLR could be effective parameters in predicting the presence of the osteoarticular involvement in patients with brucellosis.

Several studies have reported that the MPV and RDW are inflammatory markers in infectious and rheumatic diseases, as a diagnostic and therapeutic guide ${ }^{14,15}$. No statistically significant differences were reported in a study comparing the MPV and the RDW in healthy controls and patients with acute brucellosis11. Additionally, Togan et al. ${ }^{12}$ reported that the MPV and RDW in patients with osteoarticular brucellosis were compatible with the control group. In our study, no significant difference was observed between patients with and without the osteoarticular involvement $(\mathrm{P}>0.05)$, implying that these values do not play a vital role in the brucellosis diagnosis.

Correlations between CRP and the ESR and leucocyte ratios have been assessed in several studies in various subjects. He et al. ${ }^{29}$ reported that the NLR correlated significantly with CRP ( $r, 0.412 ; \mathrm{P}<0.001)$ and the ESR ( $\mathrm{r}$, $0.362 ; \mathrm{P}=0.002)$ in patients with tuberculosis spondylitis. In another study, the NLR and MLR correlated positively with CRP and the ESR in osteoarthritis, ankylosing spondylitis and rheumatoid arthritis ${ }^{31}$. To the best of our knowledge, this is the first study to analyse the correlation between the leucocyte sub-group ratio and inflammation markers and assess cut-off values in patients with osteoarticular brucellosis. In this study, a positive correlation of the NLR and MLR with the ESR and CRP was detected in the patient group. The NLR is an easier and cheaper test compared with other inflammatory biomarkers such as $\mathrm{CRP}^{32}$. Hence, leucocyte sub-group ratios could be an alternative to inflammatory markers in predicting the osteoarticular involvement in patients with brucellosis. The sensitivity and specificity of the ESR, CRP, NLR and MLR cut-off values obtained from patients with osteoarticular brucellosis should be supported with different studies. However, the retrospective nature of this study and a relatively sample size are limitations of this study.

\section{Conclusion}

The NLR, MLR, ESR and CRP values in patients with osteoarticular brucellosis were remarkably increased compared with those without any local involvement. Additionally, the NLR and MLR correlate positively with inflammatory markers. Hence, this study suggests that the NLR and MLR could be an alternative to inflammatory markers in brucellosis with the osteoarticular involvement and that the NLR, MLR, ESR and CRP could be useful parameters in estimating the clinical course of patients with brucellosis.

\section{Declaration of conflict of interest}

The authors have no conflict of interest to declare.

\section{References}

1. Young EJ. Brucella species. In: Mandell GL, ed. Principles and practice of infectious diseases. $6^{\text {nd }}$ ed. Philadelphia: Churchill Livingstone, 2005. p. 2669-72.

2. Pappas G, Akritidis N, Bosilkovski M, Tsianos E. Brucellosis. N Engl J Med. 2005; 352: 2325-2336.

3. Aktas F, Senol E, Yetkin A, Gurdogan K, Ulutan F. Clinical and laboratory findings of brucellosis in relation to disease stress. Turk Mikrobiyol Cem Derg. 1994; 24: 1649.

4. Sanaei Dashti A, Karimi A. Skeletal involvement of brucella melitensis in children: a systematic review. Iran J Med Sci. 2013; 38(4): 286-92.

5. Buzgan T, Karahocagil MK, Irmak H, Baran AL, Karsen $\mathrm{H}$, Evirgen $\mathrm{O}$, et al. Clinical manifestations and complications in 1028 cases of brucellosis: a retrospective evaluation and review of the literature. IJID. 2010; 14(6): 469- 78.

6. Bosilkovski M, Dimzova M, Grozdanovski K. Natural history of brucellosis in an endemic region in different time periods. Acta Clin Croat. 2009; 48(1): 41-6.

7. Öztürk ZA, Sayıner H, Kuyumcu ME, Yeşil Y, Savaş E, Sayiner ZA, et al. Mean platelet volume in assessment of brucellosis disease. Biomed Res India. 2012; 23: 541-6.

8. Mirsaeidi M, Peyrani P, Aliberti S, Filardo G, Bordon J, Blasi F, et al. Thrombocytopenia and thrombocytosis at time of hospitalization predict mortality in patients with community acquired pneumonia. Chest. 2010; 137: 416420.

9. Ahsen A, Ulu M S, Yuksel S, Demir K, Uysal M, Erdogan $\mathrm{M}$, et al. As a new inflmmatory marker for familial Mediterranean fever: neutrophil-to-lymphocyte ratio. Inflammation. 2013; 36: 1357-1362.

10. Boyraz I, Koc B, Boyaci A, Tutoglu A, Sarman H, Ozkan H. Ratio of neutrophil/lymphocyte and platelet/ 
lymphocyte in patient with ankylosing spondylitis that are treating with anti-TNF. Int J Clin Exp Med. 2014; 7: 29122915.

11. Togan T, Narci H, Turan H, Ciftci O, Kursun E, Arslan H. The Impact of Acute Brucellosis on Mean Platelet Volume and Red Blood Cell Distribution. Jundishapur J Microbiol. 2015; 8(2): e20039.

12. Togan T, Turan H, Narci H, Ciftci O, Kursun E, Arslan H. Predictive Value of Mean Platelet Volume and Red Blood Cell Distribution in Chronic Brucellosis. Flora. 2014; 19(1): 25-3.

13. Zareifar S, Farahmand Far MR, Golfeshan F, Cohan N. Changes in Platelet Count and Mean Platelet Volume During Infectious and Inflammatory Disease and Their Correlation With ESR and CRP. J. Clin. Lab. Anal 2014; 28: 245-248.

14. Beyazit Y, Sayilir A, Torun S, Suvak B, Yesil Y, Purnak $\mathrm{T}$, et al. Mean platelet volume as an indicator of disease severity in patients with acute pancreatitis. Clin Res Hepatol Gastroenterol. 2012; 36(2): 162-8.

15. Küçükbayrak A, Taş T, Tosun M, Aktaş G, Hakyemez IN, Mengeloğlu FZ. Erythrocytes Parameters in The Course of Brucellosis. Abant Med J. 2013;2(1):36-9.

16. Pappas G, Papadimitriou P, Akritidis N, Christou L, Tsianos EV. The new global map of human brucellosis. Lancet Infect Dis. 2006; 6: 91-9.

17. Hashemi SH, Keramat F, Ranjbar M, Mamani M, Farzam A, Jamal-Omidi S. Osteoarticular complications of brucellosis in Hamedan, an endemic area in the west of Iran. Int J Infect Dis. 2007; 11: 496-500.

18. Bozkurt F, Aslan E, Deveci O, Tekin R. Evaluation of mean platelet volume levels in patients with brucellosis. Anatol J Clin Investig. 2014; 8(3): 126-129.

19. Aktuğ-Demir N, Kolgelier S, Ozcimen S, Sumer S, Demir LS, Inkaya AC. Diagnostic clues for spondylitis in acute brucellosis. Sandi Med J. 2014; 35(8): 816-820.

20. Colmenero JD, Reguera JM, Fernández-Nebro A, CabreraFranquelo F. Osteoarticular complications of brucellosis. Ann Rheum Dis. 1991; 50: 23-26.

21. Doganay M, Mese Alp E. In: Topcu AW, ed. Infectious diseases and microbiology. 3nd ed. Istanbul: Nobel Med, 2008. p. 897-909.

22. Kahramanca S, Ozgehan G, Seker D, Gökce EL, Seker G, Tunc G, et al. Neutrophil-to-lymphocyte ratio as a predictor of acute appendicitis. Ulus Travma Acil Cerrahi Derg. 2014; 20: 19-22.

23. Kounis NG, Soufras GD, Tsigkas G, Hahalis G. White blood cell counts, leukocyte ratios, and eosinophils as inflmmatory markers in patients with coronary artery disease. Clin Appl Thromb Hemost. 2014; 21: 139-43.

24. Horne BD, Anderson JL, John JM, et al. Which white blood cell subtypes predict increased cardiovascular risk? J Am Coll Cardiol. 2005; 45: 1638-1643.

25. Imtiaz F, Shafique K, Mirza SS, Ayoob Z, Vart P, Rao $\mathrm{S}$. Neutrophil lymphocyte ratio as a measure of systemic inflammation in prevalent chronic diseases in Asian population. Int Arch Med. 2012; 5(1):2.

26. Rifaıglu EN, Bulbul Sen B, Ekiz O, Cigdem Dogramac1 A. Neutrophil to lymphocyte ratio in Behçet's disease as a marker of disease activity. Acta Dermatovenerologica Alpina, Pannonica, et Adriatica, 2014; 23(4): 65-67. 27. Hu ZD, Sun Y, Guo J, Huang YL, Qin BD, Gao Q, et al. Red blood cell distribution width and neutrophil/ lymphocyte ratio are positively correlated with disease activity in primary Sjogren's syndrome. Clinical Biochemistry. 2014; 47(18): 287-290.

28. He J, Li J, Wang Y, Hao P, Hua Q. Neutrophil-to- lymphocyte ratio(NLR) predicts mortality and adverse-outcomes aftr S T-segment elevation myocardial infarction in Chinese people. International Journal of Clinical and Experimental Pathology. 2014; 7(7): 4045-4056.

29. He Q, Tang W, Deng Y, He Y, Xie L, Qin X, et al. The diagnostic value of neutrophil-to-lymphocyte ratio and platelet-to-lymphocyte ratio in tuberculous spondylitis. Int J Clin Exp Med. 2016; 9(8): 16360-16366.

30. Aydin E, Karadag MA, Cecen K, Cigsar G, Aydın S, Demir A, et al. Association of mean platelet volume and the monocyte/lymphocyte ratio with brucella caused epididymo-orchitis. SouthEast Asian J Trop Med Public Health. 2016; 47: 450-57.

31.Yang Z, Zhang Z, Lin F, Ren Y, Liu D, Zhong R, et al. Comparisons of neutrophil-, monocyte-, eosinophiland basophil- lymphocyte ratios among various systemic autoimmune rheumatic diseases. APMIS. 2017; 125: 863-871.

32.Kang YA, Kwon SY, Yoon HI, Lee JH, Lee CT. Role of C-reactive protein and procalcitonin in differentiation of tuberculosis from bacterial community acquired pneumonia. KJIM. 2009; 24: 337-342. 